\title{
An Exact Derivation of The Quark Coupling Constant Without QCD
}

\author{
J.A.de Wet \\ Box 514,Plettenberg Bay,6600,South Africa
}

\begin{abstract}
In this note we show that the quark coupling constant $q=0.06583$ yieldsthe lattice of E6 which has been shown to map the Standard Model. Thusthere is no appeal to QCD for binding the quarks.AMS Classification Code:14J247,14K25,14M25,22E70,81V05.
\end{abstract}

Keywords:Equiharmonics,Jacobi Theta Function,QCD, Coupling Constant,E6lattice,Standard Model.

\section{Introduction}

Fig.1 is the lattice of E6 with quarks assigned to the vertices, based on a modelby Slansky[9], which has been shown to agree with the Standard Model in severalpapers summarised in[6]. The vertices are also labeled by $0, \mu, \nu$ according to anotation adopted by Coxeter [4],Section 12.3, where $0 ; \mu, \nu$ can assume the values $0,2,3$ indicating rotations $\omega$ through 120 and 240 degrees. In this way thevertices of each equilateral triangle are a rotation of 120 degrees so nucleons arebound by a rotation of quarks according to su3 color symmetry with no appealto a Strong Force. Fig.1 is not the same as that given in Ref[4] but is takenfrom an earlier reference[3] which is a torus with the leptons $\tau^{ \pm}, \nu_{\tau}$ situated inthe center dictated by the infinitesimal structure of a cubic or elliptic surface.

In this note we will see how the lattice of Fig. 1 is governed by a quarkCoupling Constant $q=0.06583$ which is close to the constant 0.118 found byDavies et al.[5] where a smaller rectangular QCD matrix is employed.

Specifically E6 $=\mathrm{CP} 3$, the complex projective 3 -space, has 3 real and 3complex dimensions so we must consider rotations which are Jacobi ThetaFunctions with a nome $\mathrm{q}=\exp (-\mathrm{i} \pi \cdot \mathrm{K} / \mathrm{K})$, where $\mathrm{K}$ and $\mathrm{iK}$ are quarter periods on the real and imaginary axes. If these are equiharmonic ,or multiplesof a fundamental frequency $f$,then $\mathrm{q}=0.06583$ [1], which is shown $\mathrm{in}[8]$ to yieldiK/K$=\operatorname{sqrt} 3 / 2=\sin 120=\sin \omega$ or $\sin 60$ that is precisely the angle in Fig.1 of thetritangent that maps the quarks and anti-quarks in an equiharmonic lattice.In this way the E6 lattice carries the coupling constant q uniting the up anddown quarks and the fundamental frequency f could well be electromagneticoccupying all of space.

For example the Jacobi Theta Function given by [2] Ch.4 is

$$
\theta_{E_{\theta}+[1]}=27 q^{4 / 3}+216 q^{10 / 3}+459 q^{16 / 3}+\ldots
$$

when the origin is moved to a deep hole, ie. a translation to include the leptons $\tau^{ \pm}, \nu_{\tau}$. Here 27 is the number of quarks and leptons of the Standard Model(alsothe number of vertices in Fig.1 together with the 3 leptons in the center) and216 is the order of the subalgebra $\left(\mathrm{su}_{3}\right)_{\text {rotation }}+\left(\mathrm{su}_{3}\right)_{\text {isospin }}+\left(\mathrm{su}_{3}\right)_{\text {color }}$ ofE6(cf.[6]).

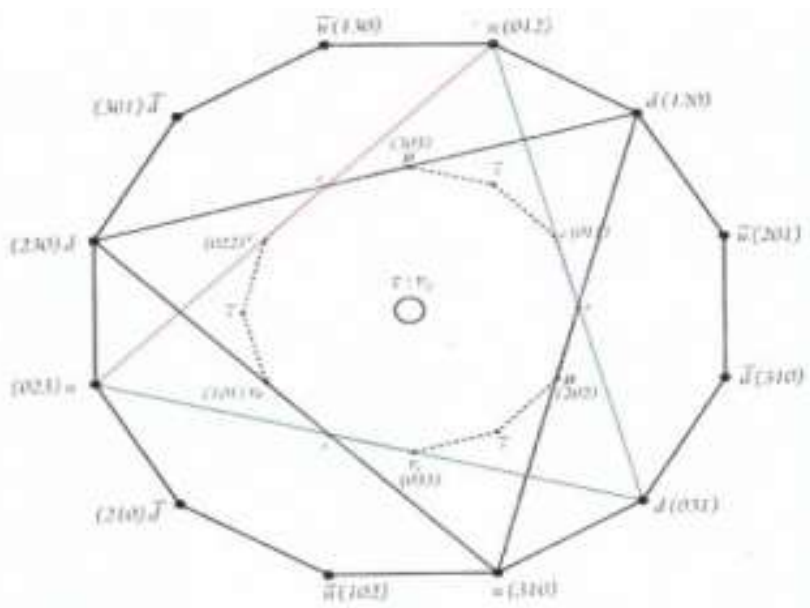

Figure 1: E6Polytope 


\section{The Equiharmonic Lattice}

Here we will provide details of of the calculation of $\omega:=120$ degrees from thenome $\mathrm{q}=\exp (-\mathrm{i} \pi \mathrm{K} / \mathrm{K})$ found in [1].Writing $\mathrm{iK} / \mathrm{K}=\mathrm{sqrt} 3 / 2$ we have the identity

$$
\ln \left(q^{-1}\right)=\pi \sqrt{3} / 2=2.7207 \Rightarrow q=0.06583
$$

for the quark coupling constant without any appeal to QCD.

\section{Conclusion}

The equiharmonic lattice of Fig. 1 may also result from $\mathrm{iK} / \mathrm{K}=$ sqrt 3 when $\omega:=60$ degrees in which case we find a possible nuclear coupling constant of 0.00433 which is the same order of magnitude as that suggested by Rees [9],Ch.4. E6 isalso the orbifold of Type II String Theory[6].

\section{References}

[1] M.Abramovitch and I.A.Stegun, Handbook of Mathematical Functions,NY,Dover (1972)

[2] J.H.Conway and N.J.A.Sloane, Sphere Packings.Lattices andGroups,,Springer NY (1993)

[3] H.S.M.Coxeter, ref3 The polytope 221 whose 27 vertices correspond to thelines of the general cubic surface, AM.MathematicalSoc.62(1940)467-486.

[4] H.S.M.Coxeter, Regular Complex Polytopes,Camb.Univ.Press(1991)

[5] C.T.H. Davies et al., Update:Accurate determination of $\alpha_{n}$ s from realisticlattice QCD, arXiv:0807.1687v3[hep-lat] Dec.(2008).

[6] J.A.de Wet, Icosahedral Symmetry:AReview,Int.Frontier Science Letters,Scipress ltd.1-8,online.

[7] J.A.de Wet, An algebraic approach to QCD and superconduction, International Mathematical Forum,v8(2013)11111117,HIKARILTD., dx.doi.org/10.12988/imf.2013.3477.

[8] J.A.de Wet, The equiharmonic lattice ofE6,online under ResearchGate.

[9] Martin Rees, Just Six Numbers, Weiden_eldandNicolson(2015)

[10] R.Slansky, Group theory for uni_ed model building, Reprinted in Unity ofForces in the Universe, Ed.ALee,WorldScienti_c(1992). 